\title{
Effects of Short-Term Aging on Asphalt Binders and Hot Mix Asphalt at Elevated Temperatures and Extended Aging Time
}

\author{
Rubben Lolly ${ }^{1}$, Waleed Zeiada ${ }^{2, * *}$, Mena Souliman ${ }^{3}$, and Kamil Kaloush ${ }^{4}$ \\ ${ }^{1}$ Street Transportation Department, City of Phoenix, 200 W. Washington St., Phoenix, Arizona \\ 85003, USA \\ ${ }^{2}$ Department of Civil and Environmental Engineering, University of Sharjah, P.O Box 27272 Sharjah, \\ UAE \\ ${ }^{3}$ Departments of Civil Engineering and Construction Management, The University of Texas at Tyler \\ 3900, University Blvd Tyler, TX 75701, USA \\ ${ }^{4}$ Department of Civil, Environmental, and Sustainable Engineering, Arizona State University \\ P.O. Box 875306, Tempe, AZ 85287-5306, USA
}

\begin{abstract}
The production process of Hot Mix Asphalt (HMA) causes a short term aging (STA) to asphalt binder due to the heating of both asphalt binder and aggregates before mixing together. Laboratory protocols are followed to simulate the STA conditions for both asphalt binders and asphalt mixtures. STA protocols expose asphalt binders or asphalt mixtures to specific aging temperature for a specific period of time to produce stiffening that is similar to that of actual production conditions. Successful construction of HMA in cold season/regions may require elevating the production temperature of HAM to achieve proper compaction of HMA layers. Producing HMA mixtures at elevated temperatures may cause further increase in the binder stiffness and negatively affect the future field performance of asphalt pavements. This negative affect can be even worse especially if it is coupled with extended exposer time increase. This study aims to investigate effect of elevated production (mixing and STA) temperatures and exposure time on the stiffening of binders and asphalt mixtures. The binder experiment involved aging of two Performance Graded (PG) binders (PG 76-16 and PG 64-22) at two different temperatures and aging durations. The asphalt mixture experiment involved the STA of asphalt mixtures produced in the laboratory at mixing and STA temperatures $25^{\circ} \mathrm{F}$ above standard practice and aging time 2 and 4 hours longer than standard practices. The effect of different aging times and temperatures was investigated by running viscosity tests on binders and dynamic modulus $\left|E^{*}\right|$ and Indirect Diametrical Strength (IDT) tests on asphalt mixtures. The results showed that increasing the mixing and STA temperatures by $25^{\circ} \mathrm{F}$ seems to have no significant effect on the asphalt mixture properties while doubling the standard STA time seems to have a significant effect on binder and asphalt mixture properties.
\end{abstract}

\footnotetext{
* Corresponding author: wzeiada@sharjah.ac.ae
} 


\section{Introduction}

Short term aging of asphalt binders refers to the hardening of asphalt that occurs during mixing, storing, transporting and placement operations. This hardening is a result of the volatilization and loss of lighter aromatic fractions from the binder due to exposure to high temperature, leaving behind larger and stiffer fraction asphaltenes [1]. The extent of aging depends mainly on type of asphalt, temperature (mixing temperature, atmospheric temperature, ground surface temperature), and time (mixing time, storage time, haul distance time, waiting time of haul trucks). The 2015 Shell Handbook recommends that the best practices for providing coating viscosity while limiting aging due to elevated temperatures is to use the minimum temperatures necessary to attain required coating viscosities [2]. It acknowledges the practice of mixing hot mix asphalt (HMA) at elevated temperatures to address the need for maintaining adequate lay down temperatures under conditions of low ambient temperatures and long hauling distances. For asphalt binders, the effect of short term aging is simulated in the laboratory by the Rolling Thin Film Oven (RTFO), which exposes the binder to continuous heat and air flow that promotes oxidation to cause aging. Asphalt binders are organic bituminous substances hence are affected by the presence of oxygen, ultra violet radiation and changes in temperature resulting in increased penetration increase in softening point and viscosity [3]. On the other hand for Hot Mix Asphalt (HMA), the short term aging in the field is simulated in the laboratory using the Short Term Oven Aging (STOA) procedure which involves heating a loose mix in a forced draft oven for 4 hours at a temperature of $135^{\circ} \mathrm{C}\left(275^{\circ} \mathrm{F}\right)$ [4]. Research conducted by the University of California at Berkeley in conjunction with Oregon State University and Austin Research Engineers, Inc. evaluated the influence of aging in the performance test result of asphalt pavements. It was concluded that 4 hours short-term aging adequately represents the effect of aging due to mix production and construction stages when the mix is in the loose state [5]. Azari (2011) investigated the effect of laboratory short-term aging conditioning on the mechanical properties of asphalt mixtures. Asphalt samples were conditioned at $145^{\circ} \mathrm{C}$ at half-hour intervals from zero to six hours and measured the dynamic modules at $4^{\circ} \mathrm{C}, 20^{\circ} \mathrm{C}, 40^{\circ} \mathrm{C}$, and $45^{\circ} \mathrm{C}$. It was concluded that for each pair of samples (aged versus un-aged) the effect of conditioning time was significant for the dynamic modulus and the minimum conditioning time to measure changes in dynamic modulus of the mixture due short-term aging conditions was 3.5 hours.

Construction of asphalt pavements involves the production of the asphalt mixtures at temperatures prescribed by asphalt binder manufactures. Placement and compaction of asphalt pavements require minimum temperatures for the construction of a quality and good performing pavement. Particularly in cooler temperature regions or seasons or areas where construction sites are farther away from asphalt production plants, the loss of temperature can, sometimes, results in the mixes arriving at the construction site with temperatures below specified lay down and compaction temperature. Such cases cause loads of asphalt mixes to be rejected causing waste of material, delays in construction scheduling and the risk of disputes resulting from delays in the completion of projects. In addition to the difficulty in achieving or maintaining compaction temperatures in cooler seasons, most agencies require minimum surface temperatures before allowing paving to occur. For example, in the 2012 Maricopa Association of Governments (MAG) uniform standard specifications for construction, a minimum surface temperature of $45^{\circ} \mathrm{F}$ and rising is required to allow paving operation to proceed. This leads to paving operations that are halted, delayed or shut down during the winter months. To overcome these delays and waste associated with constructions shut downs, contractors have proposed that hot mix asphalt be produced at higher than prescribed temperature as long as compaction densities or target air voids and binder contents are achieved in the field. However, the concern with 
elevated temperatures is that they may further increase the degree of short term aging to a level that may adversely affect the performance of the asphalt pavement. Several research studies have shown that the volatilization and oxidation are the biggest contributors to the hardening (aging) process of asphaltic binders [6]. These mechanisms are enhanced at elevated temperatures hence increasing the aged properties of the binders in the mixture. In some instances, longer hauling distance means that the hot mix asphalt will be subjected to elevated temperature for a longer period of time, possibly causing further hardening. The combined effect of elevated temperature and longer exposure time may cause excessive short term aging and adversely affect the performance of the pavement.

\section{Objectives}

The objective of this study was to investigate the combined effect of elevated temperatures and extended exposure time on short term aging properties of asphalt binders and mixtures. The objectives were achieved in two stages. The first stage involved determining the short term aging properties of the binders conduct by laboratory aging of two Arizona binders and measuring the viscosities and penetration. The second stage involved aging loose mixtures at different temperatures and aging times. The aged samples were compacted and then their dynamic modulus and indirect tensile strengths were measured.

\section{Testing program}

The testing program was divided into two main parts. The first part evaluated the short term aging effect on asphalt binders. It involved aging two performance graded (PG) virgin asphalt binders (PG 76-16 and PG 64-22) in Rolling Thin Film Oven (RTFO) using ASTM D2872 test protocol [7]. The samples were supplied by Holly Asphalt in collaboration with the Arizona Department of Transportation (ADOT). Both asphalt binders were aged at two different temperatures (standard temperature $\left(322^{\circ} \mathrm{F}\right)$ and elevated temperature $\left(350^{\circ} \mathrm{F}\right)$ ) as well as two different aging times (standard aging time (85 minutes) and prolonged aging time $(170$ minutes $))$. The aged binders were then tested to measure their rotational viscosities (ASTM D4402-06) [8], penetration (ASTM-D5-06) [9], and softening point (ASTM D36/D36M-09) [10]. Table 1 shows a summary of the testing regime used for the first stage of the study.

Table 1. Summary of testing regime and protocol for binder aging study.

\begin{tabular}{|c|c|c|c|c|c|}
\cline { 2 - 6 } \multicolumn{1}{c|}{} & \multicolumn{2}{c|}{ Aging Test } & \multicolumn{3}{c|}{ Viscosity Test } \\
\hline Binder & $\begin{array}{c}\text { RTFO Temp. } \\
\left({ }^{\circ} \text { F) }\right.\end{array}$ & $\begin{array}{c}\text { RTFO Time } \\
\text { (Minutes) }\end{array}$ & $\begin{array}{c}\text { Penetration } \\
\text { Test }\end{array}$ & $\begin{array}{c}\text { Brookfield } \\
\text { Test }\end{array}$ & $\begin{array}{c}\text { Softening Point } \\
\text { Test }\end{array}$ \\
\hline PG 64-22 & 322 & 85 & 2 replicates & 2 replicates & 2 replicates \\
\hline PG 64-22 & 350 & 85 & 2 replicates & 2 replicates & 2 replicates \\
\hline PG 64-22 & 350 & 175 & 2 replicates & 2 replicates & 2 replicates \\
\hline PG76-16 & 322 & 85 & 2 replicates & 2 replicates & 2 replicates \\
\hline PG76-16 & 350 & 85 & 2 replicates & 2 replicates & 2 replicates \\
\hline PG76-16 & 350 & 175 & Not Tested & Not Tested & Not Tested \\
\hline
\end{tabular}


The second part of this study involved the aging of laboratory produced asphalt mixtures in their loose state before compacted into gyratory plugs using the PG 64-22 binder. The loose asphalt mixtures were aged at standard aging temperature $\left(275^{\circ} \mathrm{F}\right)$ and elevated temperature $25^{\circ} \mathrm{F}$ above the standard STA temperature. The STA time was also increased by 2 and 4 hours longer than the standard practice of 4 hours in the. The prepared testable specimens at each STA temperature and time combination were then tested using the Dynamic Modulus $\left|E^{*}\right|$ test (AASHTO TP-62-07) [11] and the Indirect Tensile Strength (IDT) test (ASTM D6931-07) [12]. Table 2 presents a summary of the testing matrix and manufactured specimens. Figure 1 shows a summary of the experimental Program

Table 2. Summary of mixture aging matrix and testing regime.

\begin{tabular}{|c|c|c|c|c|c|c|}
\hline Condition & \begin{tabular}{|c} 
Mixing \\
Temperature
\end{tabular} & $\begin{array}{c}\text { Aging } \\
\text { Temperature }\end{array}$ & Aging Time & $\begin{array}{c}\text { Sample } \\
\text { ID }\end{array}$ & $\begin{array}{c}\text { Test } \\
\text { Performed }\end{array}$ & $\begin{array}{l}\text { \% Air } \\
\text { Voids }\end{array}$ \\
\hline \multirow{12}{*}{ 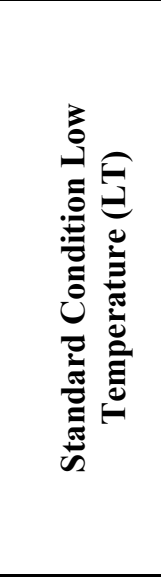 } & \multirow{12}{*}{$305^{\circ} \mathrm{F}$} & \multirow{12}{*}{$275^{\circ} \mathrm{F}$} & \multirow{4}{*}{4 hours } & AG4L1 & $\left|E^{*}\right|$ & $6.83 \%$ \\
\hline & & & & AG4L2 & IDT & $6.27 \%$ \\
\hline & & & & AG4L3 & $\left|E^{*}\right|$ & $7.30 \%$ \\
\hline & & & & AG4L4 & $\left|E^{*}\right|$ & $6.74 \%$ \\
\hline & & & \multirow{4}{*}{6 hours } & AG6L1 & $\left|E^{*}\right|$ & $6.85 \%$ \\
\hline & & & & AG6L2 & IDT & $6.43 \%$ \\
\hline & & & & AG6L3 & $\left|E^{*}\right|$ & $7.19 \%$ \\
\hline & & & & AG6L4 & $\left|E^{*}\right|$ & $6.15 \%$ \\
\hline & & & \multirow{4}{*}{8 hours } & AG8L1 & $\left|E^{*}\right|$ & $6.70 \%$ \\
\hline & & & & AG8L2 & IDT & $6.39 \%$ \\
\hline & & & & \begin{tabular}{|l} 
AG8L33 \\
\end{tabular} & $\left|E^{*}\right|$ & $7.72 \%$ \\
\hline & & & & AG8L4 & $\left|E^{*}\right|$ & $6.94 \%$ \\
\hline \multirow{12}{*}{ 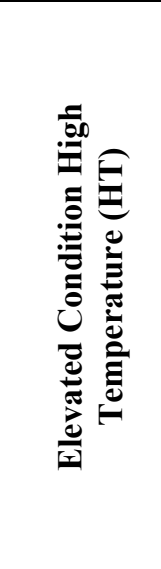 } & \multirow{12}{*}{$330^{\circ} \mathrm{F}$} & \multirow{12}{*}{$300^{\circ} \mathrm{F}$} & \multirow{4}{*}{4 hours } & AG4H1 & $\left|E^{*}\right|$ & $7.60 \%$ \\
\hline & & & & AG4H2 & IDT & $6.18 \%$ \\
\hline & & & & \begin{tabular}{|l} 
AG4H3 \\
\end{tabular} & $\left|E^{*}\right|$ & $6.49 \%$ \\
\hline & & & & AG4H4 & $\left|E^{*}\right|$ & $7.25 \%$ \\
\hline & & & \multirow{4}{*}{6 hours } & AG6H1 & $\left|E^{*}\right|$ & $7.54 \%$ \\
\hline & & & & AG6H2 & IDT & $6.28 \%$ \\
\hline & & & & \begin{tabular}{|l} 
AG6H33 \\
\end{tabular} & $\left|E^{*}\right|$ & $7.68 \%$ \\
\hline & & & & AG6H4 & $\left|E^{*}\right|$ & $7.20 \%$ \\
\hline & & & \multirow{4}{*}{8 hours } & AG8H1 & $\left|E^{*}\right|$ & $7.19 \%$ \\
\hline & & & & AG8H2 & IDT & $6.41 \%$ \\
\hline & & & & AG8H3 & $\left|E^{*}\right|$ & $7.25 \%$ \\
\hline & & & & AG8H4 & $\left|E^{*}\right|$ & $7.80 \%$ \\
\hline
\end{tabular}




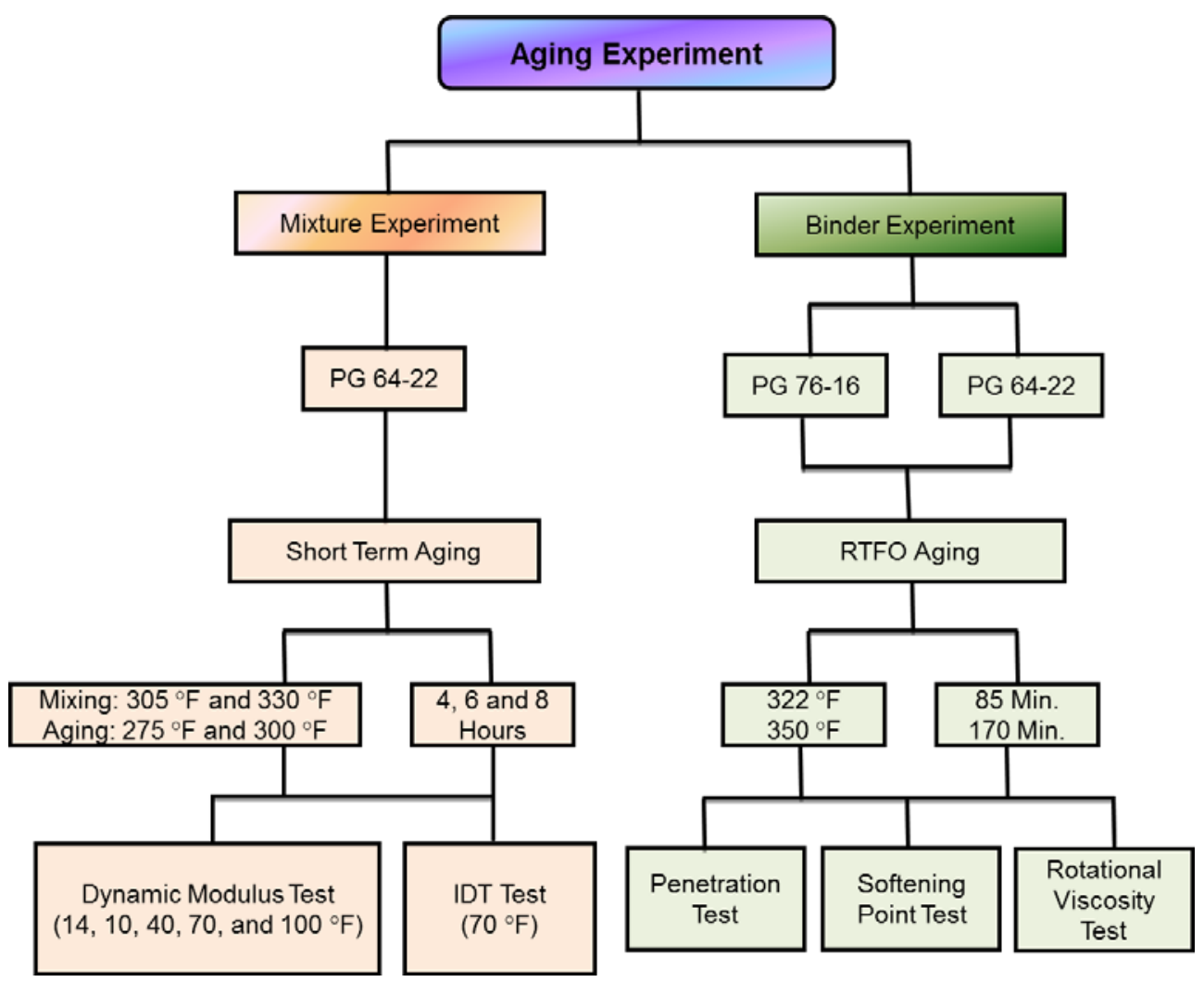

Fig. 1. Summary of the experimental program.

\section{Test methods and results of asphalt binders}

RTFO aging was first performed on samples of PG 76-16 and PG 64-22 binders at $322^{\circ} \mathrm{F}$ for 85 minutes each as prescribed in ASTM D2872. A second set of samples were aged in the RTFO at $350^{\circ} \mathrm{F}$ for 85 minutes. In order to evaluate the effect of aging time in the RTFO, a third batch of PG 64-22 binder was aged in the RTFO at $350^{\circ} \mathrm{F}$ for 175 minutes doubling the prescribed time specified in the ASTM D2872.

Upon completion of the STA process in the RTFO, three test methods were conducted to measure the consistency of asphalt binders at different STA temperatures and time. These test methods are penetration, softening point, and Brookfield rotational viscosity. Two replicates samples of each aged asphalt binders were used for each test method. The Brookfield rotational viscosity test measures viscosity directly while the softening point and penetration tests measure viscosity indirectly using correlations from the test result.

The penetration measurements were converted to viscosity using the following equation developed by Mirza and Witczak in 1995 [1]:

$$
\log \eta=10.5012-2.2601 * \log (\text { pen })+0.00389 *(\log (\text { pen }))^{2}
$$

Where $\eta$ is the viscosity in poise (P) and Pen is the measured average penetration loading in $0.10 \mathrm{~mm}$. 
For the softening point test, the temperature at which the deformed sample touches the brass shelf at the bottom of the assembly shelf is recorded as the softening point of the binder. The softening point is the temperature at which the binder has a viscosity of 13,000 poise (ASTM D36/D36M-09). Brookfield viscosity was conducted at $250^{\circ} \mathrm{F}, 300^{\circ} \mathrm{F}, 350^{\circ} \mathrm{F}$ and $375^{\circ} \mathrm{F}$ while penetration test was conducted using conditioned binder sample at temperature of $77^{\circ} \mathrm{F}$.

Table 3 shows the effect of RTFO temperature and time on the viscosity of the PG 6422 binder. The first section of Table 3 compares the viscosity values from both rotational viscometer and penetrations test as well the softening point temperature of the base RTFO temperature $\left(322^{\circ} \mathrm{F}\right)$ to the elevated RTFO temperature $\left(350^{\circ} \mathrm{F}\right)$. Changes in viscosity are measured relative to the base viscosity. The results indicate an average of $9 \%$ increase in the overall Brookfield viscosity, $18 \%$ increase in the penetration viscosity measured at $77^{\circ} \mathrm{F}$, and $4 \%$ increase of the softening point due to increased short term aging temperature from $322^{\circ} \mathrm{F}$ to $350^{\circ} \mathrm{F}$. The Brookfield rotational viscosity changes show also that the PG 64-22 binder becomes less susceptible to temperature changes when aged at $350^{\circ} \mathrm{F}$ compared to the base RTFO temperature. The second section of Table 3 compares the viscosity values and the softening point of the PG 64-22 binder at RTFO standard exposure time ( 85 minutes) and the extended aging time (170 minutes) when both aged at $350^{\circ} \mathrm{F}$. Changes in viscosity due to extended short-term aging time of 175 minutes are measured relative to the base time viscosity at the elevated temperature. The results indicate an average increase of $99 \%$ in the overall Brookfield rotational viscosity, $92 \%$ increase in the penetration viscosity and $10 \%$ increase softening point temperature due to increased aging time from 85 minutes to 175 minutes. In addition, it was observed that increasing the short term aging time increased the Brookfield rotational viscosity values across all temperatures which was different than the trend observed for elevated temperature case (higher effect at lower temperatures compared to higher temperatures). The test results showed also that the effect of doubling the RTFO aging time on the viscosity values is more substantial that the effect of elevated RTFO temperature. Based on this observation, the time factor seems more critical than the worry about increasing the mixing temperature by $25{ }^{\circ} \mathrm{F}$ to achieve proper compaction levels in the cold paving seasons.

Table 4 shows effect of RTFO temperature on the viscosity and softening point of the PG $76-16$ binder $\left(322^{\circ} \mathrm{F}\right.$ versus $\left.350^{\circ} \mathrm{F}\right)$ at 85 minutes standard exposure time. As previously mentioned, the effect of increasing aging time was not studied for the PG 76-16 binder. Viscosities measured at the base temperature represent the base viscosity hence changes in viscosity are measured relative to the base viscosity. The results indicate an average of $4 \%$ increase in the overall Brookfield viscosity, $60 \%$ increase in the penetration viscosity measured at $77^{\circ} \mathrm{F}$ and $4 \%$ increase in softening point due to increased short term RTFO aging temperature from $322^{\circ} \mathrm{F}$ to $350^{\circ} \mathrm{F}$ at 85 minutes aging time. It can be clearly observed that effect of increasing the RTFO temperature on the Brookfield rotational viscosity and softening point is higher for the PG 64-22 binder when compared to the PG 76-22 binder as expected. However, the penetration viscosity results of the PG 76-22 showed an opposite trend where the increase in penetration viscosity was $60 \%$ for the PG 70-16 binder which is three times higher than the PG 64-22 binder. However this result was not expected, it demonstrates an anomaly in the general trend of all the test results and may be due to error during testing. 
Table 3. Comparison of PG 64-22 test results

\begin{tabular}{|c|c|c|c|c|c|c|}
\hline \multirow[t]{2}{*}{ Consistency Test } & \multicolumn{4}{|c|}{ Brookfield Viscosity, (cP) } & $\begin{array}{c}\text { Penetration } \\
\text { Viscosity, }(\mathrm{cP})\end{array}$ & \multirow{2}{*}{$\begin{array}{l}\text { Softening } \\
\text { point, }\left({ }^{\circ} \mathrm{F}\right)\end{array}$} \\
\hline & $250^{\circ} \mathrm{F}$ & $300^{\circ} \mathrm{F}$ & $350^{\circ} \mathrm{F}$ & $375^{\circ} \mathrm{F}$ & $77^{\circ} \mathrm{F}$ & \\
\hline \multicolumn{7}{|c|}{85 minutes RTFO aging time } \\
\hline $322^{\circ} \mathrm{F}$ RTFO & 1,410 & 307 & 94.5 & 60.9 & $4.14 \mathrm{E}+09$ & 129.0 \\
\hline $350^{\circ} \mathrm{F}$ RTFO & 1,770 & 338 & 99.2 & 58.6 & $4.89 \mathrm{E}+09$ & 134.2 \\
\hline \multirow{2}{*}{ Percent Change } & $26 \%$ & $10 \%$ & $5 \%$ & $-4 \%$ & \multirow{2}{*}{$18 \%$} & \multirow{2}{*}{$4 \%$} \\
\hline & & Avera & $e=9 \%$ & & & \\
\hline \multicolumn{7}{|c|}{170 minutes RTFO aging time } \\
\hline $350^{\circ} \mathrm{F}$ RTFO & 1,770 & 338 & 99.2 & 58.6 & $4.89 \mathrm{E}+09$ & 134.0 \\
\hline $350^{\circ} \mathrm{F}$ RTFO & 3,380 & 674 & 196.8 & 121.0 & $9.41 \mathrm{E}+09$ & 148.1 \\
\hline \multirow{2}{*}{ Percent Change } & $90 \%$ & $100 \%$ & $98 \%$ & $107 \%$ & \multirow{2}{*}{$92 \%$} & \multirow{2}{*}{$10 \%$} \\
\hline & & Averag & $=99 \%$ & & & \\
\hline
\end{tabular}

Table 4. Comparison of PG 76-16 test results

\begin{tabular}{|c|c|c|c|c|c|c|}
\hline \multirow{2}{*}{ Consistency Test } & \multicolumn{7}{|c|}{ Brookfield Viscosity, $(\mathrm{cP})$} & $\begin{array}{c}\text { Penetration } \\
\text { Viscosity, (cP) }\end{array}$ & \multirow{2}{*}{$\begin{array}{c}\text { Softening } \\
\text { point, }\left({ }^{\circ} \mathrm{F}\right)\end{array}$} \\
\cline { 2 - 6 } & $250^{\circ} \mathrm{F}$ & $300^{\circ} \mathrm{F}$ & $350^{\circ} \mathrm{F}$ & $375^{\circ} \mathrm{F}$ & $77^{\circ} \mathrm{F}$ & \\
\hline $322^{\circ} \mathrm{F}$ RTFO & 3,760 & 654 & 183 & 106 & $6.70 \mathrm{E}+09$ & 147 \\
\hline $350^{\circ} \mathrm{F}$ RTFO & 3,940 & 679 & 188 & 109 & $1.07 \mathrm{E}+10$ & 169 \\
\hline \multirow{2}{*}{ Percent Change } & $5 \%$ & $4 \%$ & $3 \%$ & $3 \%$ & $60 \%$ & $2 \%$ \\
\cline { 2 - 3 } & \multicolumn{7}{|c|}{ Average $=4 \%$} & & $2 \%$ \\
\hline
\end{tabular}

\section{Materials and specimen preparation}

The purpose of the study was to determine the effect of short term aging due to elevated STA temperatures and extended STA time. The typical production mix temperatures of the PG 64-22 binder is $305^{\circ} \mathrm{F}$. The standard laboratory short term aging temperature is $275^{\circ} \mathrm{F}$ for a standard time of 4 hours to simulate aging that occurs at typical production temperatures. In order to simulate the effect of a $25^{\circ} \mathrm{F}$ higher production temperature, laboratory mixing and aging temperatures of $330^{\circ} \mathrm{F}$ and $300^{\circ} \mathrm{F}$ respectively were used for this study. Samples mixed and aged at standard conditions were designated as (LT), and samples that were mixed and aged at $330^{\circ} \mathrm{F}$ were designated as (HT). To investigate the effect of extended STA time on the laboratory performance of asphalt mixture, STA time of 6 and 8 hours were considered beside the standard STA time (4 hours).

A dense graded ADOT 19mm MAG Superpave high traffic mix with PG 64-22 binder was used for this part of the study. Binder content for the mix was $4.5 \%$. Before samples were prepared, the aggregates were first oven dried, sieved and batched. Before mixing, the batched aggregates were preheated overnight to ensure drying at the respective mixing temperatures. The binders were also preheated two hours before introduced to the preheated aggregates and properly mixed. The asphalt mixtures were then short term aged and compacted with a Servopac Gyratory Compactor into a 6 inches $(150 \mathrm{~mm})$ diameter molds. Test specimens, 4 inches $(100 \mathrm{~mm})$ in diameter by 6 inches $(150 \mathrm{~mm})$ high, were cored from the center of the gyratory specimens. A total of 24 test specimens samples were manufactured for this study: 12 specimens for each LT and HT conditions. For LT 
conditions, 4 specimens were manufactured at STA times of 4 hours, 6 hours and 8 hours for total of 12 specimens. Similarly 12 specimens were manufactured for HT conditions at the same STA times. Three specimens from each group with the same STA temperature and time were used for $\left|E^{*}\right|$ testing in accordance with AASHTO TP 62-07. One specimen from each group was split into two halves and used for IDT testing.

\section{Test methods and results of asphalt mixtures}

\subsection{Dynamic modulus test}

The $\left|E^{*}\right|$ tests were conducted per AASHTO TP 62-07 at five temperatures 14, 40, 70, 100, and $130{ }^{\circ} \mathrm{F}\left(-10,4.4,21.1,37.8,54.4^{\circ} \mathrm{C}\right)$ and six load frequencies: $25,10,5,1,0.5$ and 0.1 Hz. The stress levels were varied with the frequency and temperature to keep the measured on-specimen strain amplitude below $150 \mu \varepsilon$. A dynamic haversine stress (continuous wave) was applied and measured through a load cell, whereas, the deformations were measured using spring-loaded Linear Variable Differential Transducers (LVDTs). The LVDTs were secured in-place using brackets and studs glued onto the specimens. Guide rods were added to the instrumentation to ensure good alignment.

Using the $\left|E^{*}\right|$ values obtained in this study, $\left|E^{*}\right|$ master-curves were constructed for all LT and HT asphalt mixtures (three asphalt mixtures for each case). The master modulus curve can be mathematically modelled by a sigmoidal function described as:

$$
\log |E *|=\delta+\frac{\alpha}{1+\frac{1}{e^{\beta+\gamma\left(\log f_{r}\right)}}}
$$

where:

$f_{r} \quad=$ reduced frequency of loading

$\delta \quad=$ minimum value of $\left|E^{*}\right|$

$\delta+\sigma=$ maximum value of $\left|E^{*}\right|$

$\beta, \gamma=$ parameters describing the shape of the sigmoidal function

Results from $\left|E^{*}\right|$ tests were used to predict the $\left|E^{*}\right|$ master curves for each combination of STA mixing temperature and time. The results were also used to analyse the significance of STA mixing temperature and STA time on the $\left|E^{*}\right|$ values of asphalt mixture.

\subsubsection{Effect of temperature on predicted $\left|E^{*}\right|$}

Figure 2 compares $\left|E^{*}\right|$ master-curves of asphalt mixtures due to elevated STA temperature (LT and HT aging conditions) with the same STA times of 4, 6, and 8 hours. Results presented in Figure 2 showed that for asphalt mixtures with STA times of 4 and 6 hours that there is no noticeable difference in $\left|E^{*}\right|$ master-curves while difference seems noticeable for the 8 hours STA conditions. For all the three STA time conditions, the $\left|E^{*}\right|$ values at very low frequencies (low vehicular speeds) and high testing temperatures are higher for the asphalt mixtures aged at the standard LT conditions when compared to those aged at elevated HT condition. On the other hand, at high frequency and low temperatures, $\left|E^{*}\right|$ values at high frequencies (high vehicular speeds) and low testing temperatures are lower for the asphalt mixtures aged at the HT conditions when compared to those aged at standard LT condition. In general, it seems that a $25^{\circ} \mathrm{F}$ increase in mixing and STA temperatures 
does not affect the $\left|E^{*}\right|$ values remarkably except for the 8 hours STA condition specifically at low test frequencies and intermediate and high test temperatures.
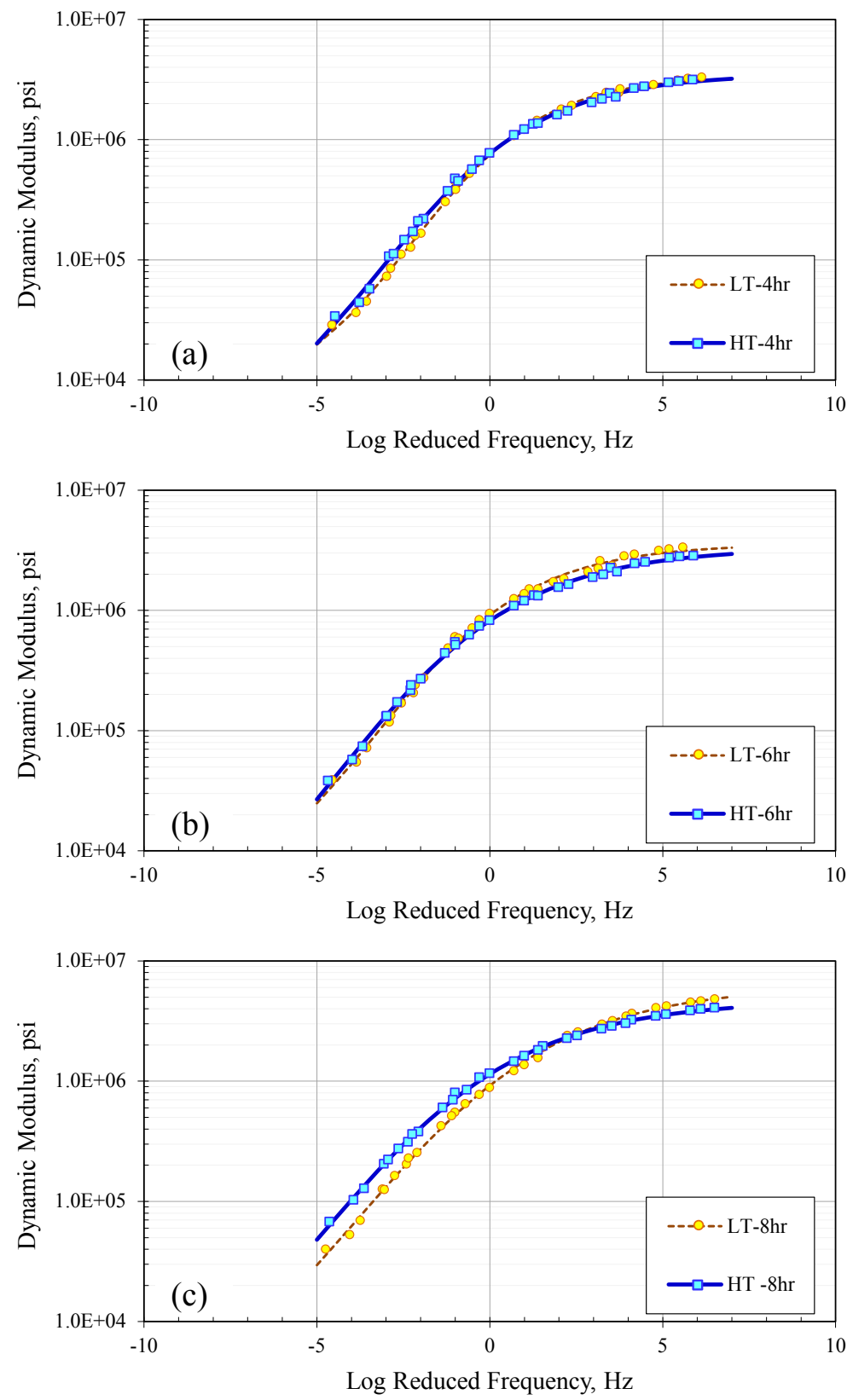

Fig. 2. Effect of mixing and STA temperatures on $\left|E^{*}\right|$ master-curve: (a) 4hours STA time; (b) 6 hours STA time; (a) 8 hours STA time. 
To determine the significance of increasing the mixing and STA temperatures by $25^{\circ} \mathrm{F}$ on the $\left|E^{*}\right|$ values, a paired t-test statistical analysis was used. The statistical analyses used a paired t-distribution for two variables. The Paired t-test assumed a null hypothesis that the increase in mixing temperature and STA temperature did not cause significant changes in the $\left|E^{*}\right|$ values asphalt mixtures. The critical assumptions of the analysis were that the mean values of the two samples were unknown and equal. In addition, a significance level $\alpha$ of $5 \%$ was assumed, and the acceptance criterion for a given hypothesis was when $\mathrm{t}_{\text {critical }} \leq \mathrm{t}$ stat $\alpha / 2, v$ where $v$ is the degree of freedom.

Table 5 includes the statistical hypothesis analysis results using the paired t-test. The results of the statistical hypothesis testing indicated that there is no significant difference between $\left|E^{*}\right|$ values of asphalt mixtures when mixing and STA temperatures increased by $25^{\circ} \mathrm{F}$ for STA time of 4 and 6 hours. However for the 8 hours STA condition, the statistical analysis showed that there is a significant difference between $\left|E^{*}\right|$ values due to elevated mixing and STA temperature. Considering a standard STA time of 4 hours, increasing the mixing and STA temperatures by $25^{\circ} \mathrm{F}$ seems to has no significant effect on the $\left|E^{*}\right|$ values measured at all test temperatures and frequencies.

Table 5. Results of Paired t-test for the effect of elevated mixing and STA temperatures on $\left|E^{*}\right|$ values at $95 \%$ confidence level.

\begin{tabular}{|c|c|c|c|c|c|c|}
\hline \multirow{3}{*}{ Paired T-Test Results } & \multicolumn{6}{|c|}{ Short Term Aging Time } \\
\hline & \multicolumn{2}{|c|}{4 Horus } & \multicolumn{2}{|c|}{6 Horus } & \multicolumn{2}{|c|}{8 Horus } \\
\hline & $\mathbf{L T}$ & HT & $\mathbf{L T}$ & HT & LT & HT \\
\hline Mean, $\boldsymbol{\mu}$ & 1259 & 1208 & 1311 & 1158 & 1717 & 1652 \\
\hline Std Dev, $\sigma$ & 1153 & 1065 & 1105 & 954 & 1654 & 1359 \\
\hline Degrees of Freedom, $v$ & \multicolumn{2}{|c|}{29} & \multicolumn{2}{|c|}{29} & \multicolumn{2}{|c|}{29} \\
\hline t Sta. $\alpha / 2, v$ & \multicolumn{2}{|c|}{2.397} & \multicolumn{2}{|c|}{5.506} & \multicolumn{2}{|c|}{1.077} \\
\hline t Critical Two-Tail & \multicolumn{2}{|c|}{2.045} & \multicolumn{2}{|c|}{2.045} & \multicolumn{2}{|c|}{2.045} \\
\hline$P(T<=t)$ Two-Tail & \multicolumn{2}{|c|}{0.023} & \multicolumn{2}{|c|}{$6.22 \mathrm{E}-06$} & \multicolumn{2}{|c|}{0.291} \\
\hline $\begin{array}{c}\text { Statistical Diff. Between } \\
\text { Sample Means }\end{array}$ & \multicolumn{2}{|c|}{ INSIGNIFICANT } & \multicolumn{2}{|c|}{ INSIGNIFICANT } & \multicolumn{2}{|c|}{ SIGNIFICANT } \\
\hline
\end{tabular}

\subsubsection{Effect of STA time on predicted $\left|E^{*}\right|$}

In order to assess the effect of increasing short-term aging time on the properties of the asphalt mixture, the $\left|E^{*}\right|$ master curves of mixtures with STA time of 4, 6 and 8 hours were compared. In general, the results showed for both LT and HT conditions that increasing the STA time increases the $\left|E^{*}\right|$ values at almost all test temperatures and frequencies. It can be also observed for both LT and HT conditions that increasing the STA time by 2 hours increases the $\left|E^{*}\right|$ values more at low frequencies and high temperatures values, while increasing the STA time by 4 hours increase the $\left|E^{*}\right|$ values at all test frequencies and temperatures. 

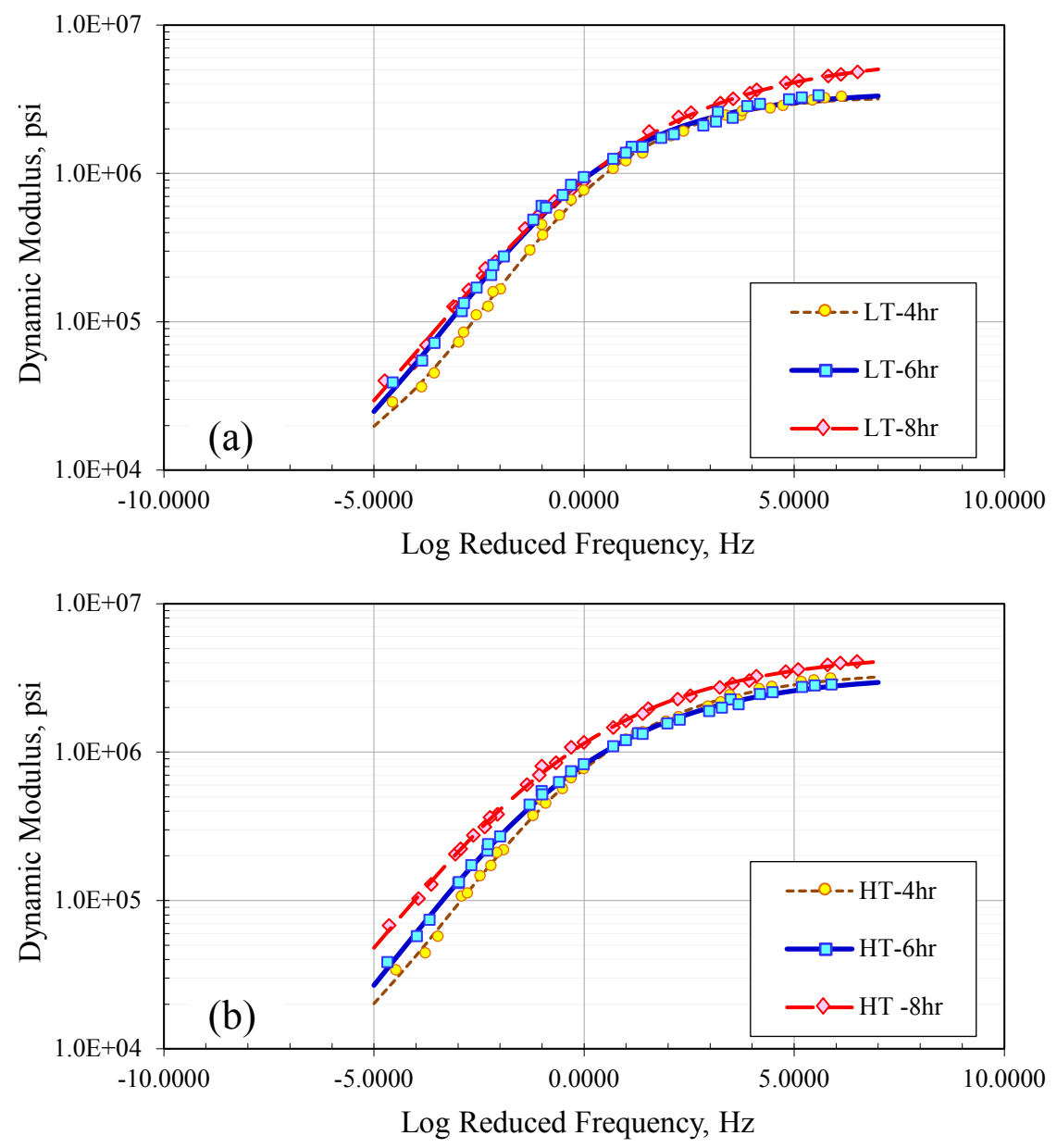

Fig. 3. Effect of STA time on $\left|E^{*}\right|$ master-curve: (a) LT aging condition; (b) HT aging condition.

Statistical hypothesis analysis was also conducted using the paired t-test to investigate the significance of increasing the STA time by 2 and 4 hours above a standard STA time of 4 hours on the $\left|E^{*}\right|$ values. Table 6 includes the statistical hypothesis analysis results using the t-paired test. The results of the statistical hypothesis testing indicated that there is no significant difference between $\left|E^{*}\right|$ values of asphalt mixtures by increasing the STA time by 2 hours, while increasing the STA time by 4 hours significantly increase the $\left|E^{*}\right|$ values. 
Table 6. Results of Paired t-test for the effect of extended STA time on $\left|E^{*}\right|$ values at $95 \%$ confidence level.

\begin{tabular}{|c|c|c|c|c|c|c|c|c|}
\hline \multirow{3}{*}{ Paired T-Test Results } & \multicolumn{8}{|c|}{ Mixing and STA Temperatures } \\
\hline & \multicolumn{4}{|c|}{ LT Condition } & \multicolumn{4}{|c|}{ HT Condition } \\
\hline & $\begin{array}{c}4 \\
\text { Horus } \\
\end{array}$ & \begin{tabular}{|c|}
6 \\
Horus
\end{tabular} & $\begin{array}{c}4 \\
\text { Horus }\end{array}$ & $\begin{array}{c}8 \\
\text { Horus }\end{array}$ & $\begin{array}{c}4 \\
\text { Horus } \\
\end{array}$ & $\begin{array}{c}6 \\
\text { Horus }\end{array}$ & $\begin{array}{c}4 \\
\text { Horus }\end{array}$ & $\begin{array}{c}8 \\
\text { Horus }\end{array}$ \\
\hline Mean, $\boldsymbol{\mu}$ & 1259 & 1311 & 1311 & 1158 & 1208 & 1158 & 1208 & 1652 \\
\hline Std Dev, $\sigma$ & 1153 & 1105 & 1105 & 954 & 1065 & 954 & 1065 & 1359 \\
\hline Degrees of Freedom, v & \multicolumn{2}{|c|}{29} & \multicolumn{2}{|c|}{29} & \multicolumn{2}{|c|}{29} & \multicolumn{2}{|c|}{29} \\
\hline t Sta. $\alpha / 2, v$ & \multicolumn{2}{|c|}{-2.366} & \multicolumn{2}{|c|}{-4.846} & \multicolumn{2}{|c|}{-2.347} & \multicolumn{2}{|c|}{-8.143} \\
\hline t Critical Two-Tail & \multicolumn{2}{|c|}{2.045} & \multicolumn{2}{|c|}{2.045} & \multicolumn{2}{|c|}{2.045} & \multicolumn{2}{|c|}{2.045} \\
\hline $\mathbf{P}(\mathbf{T}<=t)$ Two-Tail & \multicolumn{2}{|c|}{0.025} & \multicolumn{2}{|c|}{$3.89 \mathrm{E}-05$} & \multicolumn{2}{|c|}{0.026} & \multicolumn{2}{|c|}{$5.59 \mathrm{E}-09$} \\
\hline $\begin{array}{c}\text { Statistical Diff. } \\
\text { Between Sample } \\
\text { Means }\end{array}$ & \multicolumn{2}{|c|}{ INSIGNIFICANT } & \multicolumn{2}{|c|}{ SIGNIFICANT } & \multicolumn{2}{|c|}{ INSIGNIFICANT } & \multicolumn{2}{|c|}{ SIGNIFICANT } \\
\hline
\end{tabular}

\subsection{Indirect diametrical test}

The main purpose of the test is to determine the tensile strength of the tested specimens. The test can be performed on specimens compacted by the super pave gyratory compactor by cutting cored specimen into two or three parts. In this study, the cored specimens were cut into three parts each is 4 inches $(100 \mathrm{~mm})$ diameter by 2 inches $(50 \mathrm{~mm})$ height. In this test, a specimen of 4 inches $(100 \mathrm{~mm})$ diameter and not more than 2.5 inches $(63 \mathrm{~mm})$ thickness used. Before testing, all test specimens were conditioned for at least 2 hours at a target test temperature of $70{ }^{\circ} \mathrm{F}\left(21.1^{\circ} \mathrm{C}\right)$. The test specimen was then placed on a loading strip and a load with a maximum deformation rate of $2.00 \pm 0.15 \mathrm{in} / \mathrm{min}(50 \pm 5 \mathrm{~mm} / \mathrm{min})$ is slowly applied across its diametric vertical plane at a constant loading rate until failure. The peak load is then recorded $(\mathrm{P})$ and used to calculate the indirect tensile strength $\left(S_{t}\right)$ and the fracture energy $\left(G_{f}\right)$ of the specimen. Figure 4 shows the set up for the IDT test and the accumulation of fracture energy during IDT Testing.

$$
S_{t}=\frac{2 P}{\pi t d}
$$

where:

$\mathrm{d}=$ the diameter of the specimen

$\mathrm{P} \quad=$ the maximum applied load

$\mathrm{t}=$ the thickness of the test specimen or core 

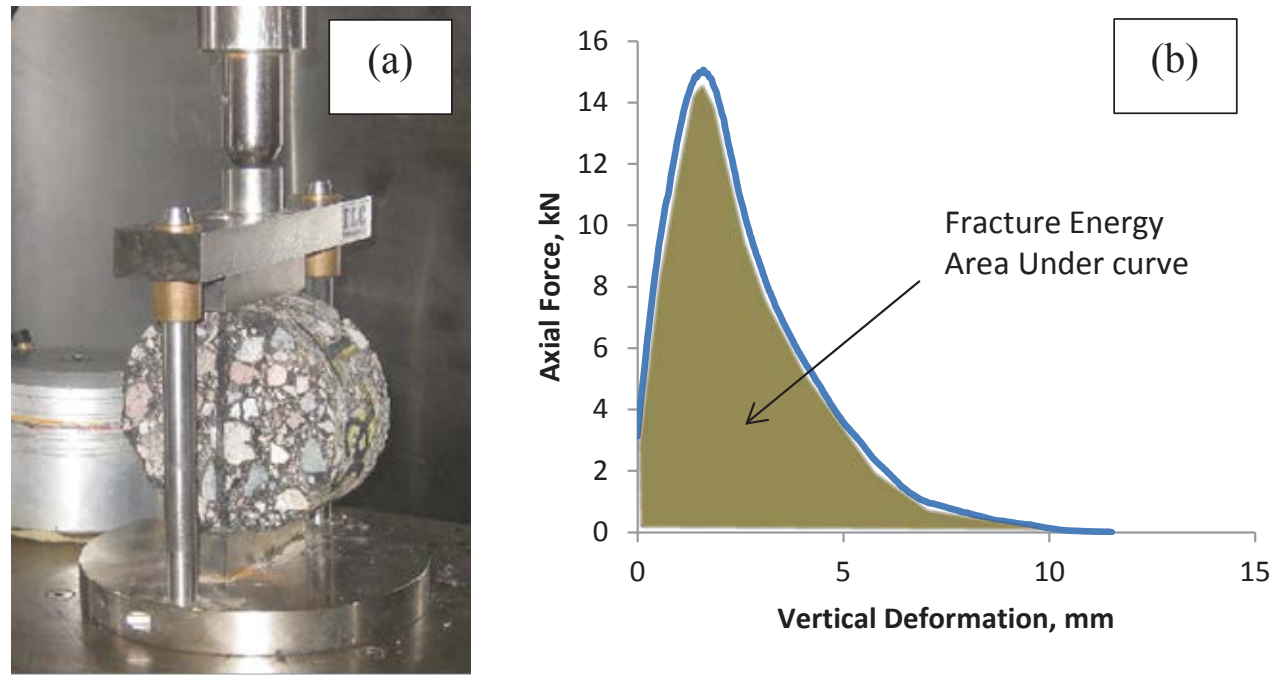

Fig. 4. (a) IDT test setup; (b) Fracture energy accumulation during IDT test.

A summary of the IDT test results are shown in Table 7 including the average values of IDT strength and fracture energy till failure as well as the variability between replicates expressed by the coefficient of variation percent (C.V.). Graphical representations and comparison of the IDT strength and fracture energy are shown in Figure 5 and Figure 6 respectively at different mixing and STA conditions. The IDT test results presented in Figure 5-a showed that increasing the production temperature of asphalt mixtures by $25^{\circ} \mathrm{F}$ increases the tensile strength for the 6 hours and 8 hours STA time. However, for a standard STA time of 4 hours, there was no clear increase in the IDT tensile strength due to the mixing and STA temperature increases. It can be observed also from Figure 5-b that extending the STA time by 2 and 4 hours increases the IDT strength when compared to the standard STA time case (4 hours).

Figure 6 showed that effect of mixing and STA conditions on the fracture energy. Increasing the production temperature as well as the STA time increases the stiffness of the asphalt mixtures which expected to decrease the fracture energy by increasing the brittleness of the asphalt mixture and decreases the vertical deformation during the test. This means decreasing the area under the load-deformation curve and decreasing the fracture energy value. Figure 6-a showed that increasing the mixing and STA temperatures by $25^{\circ} \mathrm{F}$ decrease the fracture energy for both 6 hours and 8 hours STA time conditions. For the 6 hours STA time case, there was no obvious decrease in the fracture energy for the elevated mixing and STA temperature case. On the other hand, Figure 6-b showed that increasing the STA time by 2 and 4 hours decreases the fracture energy values compared to asphalt mixtures produced at standard production temperature.

Considering the standard STA time (4 hours), it seems that there is no noticeable effect of increasing the production temperature of asphalt mixtures by $25^{\circ} \mathrm{F}$ on the IDT tensile strength or the fracture energy values. 
Table 7. Summary of IDT test results.

\begin{tabular}{|c|c|c|c|c|c|c|c|}
\hline \multicolumn{2}{|c|}{ STA Conditions } & \multirow{2}{*}{$\begin{array}{l}\text { Specimen } \\
\text { ID }\end{array}$} & \multirow{2}{*}{$\begin{array}{c}\text { Average } \\
\text { Air Voids } \\
(\%)\end{array}$} & \multicolumn{2}{|c|}{$\begin{array}{l}\text { IDT Strength } \\
(\mathrm{psi})\end{array}$} & \multicolumn{2}{|c|}{$\begin{array}{l}\text { Fracture Energy } \\
\left(\text { in.lb } / \mathrm{in}^{2}\right)\end{array}$} \\
\hline Temp. & Time & & & Average & C.V.\% & Average & C.V.\% \\
\hline \multirow{3}{*}{ LT } & 4 hours & LT-4Hrs & 6.27 & 233 & 2.3 & 46.96 & 4.6 \\
\hline & 6 hours & LT-6Hrs & 6.43 & 254 & 4.9 & 44.07 & 10.6 \\
\hline & 8 hours & LT-8Hrs & 6.39 & 243 & 3.7 & 44.22 & 11.8 \\
\hline \multirow{3}{*}{ HT } & 4 hours & HT-4Hrs & 6.28 & 231 & 3.8 & 46.30 & 16.2 \\
\hline & 6 hours & HT-6Hrs & 6.18 & 278 & 1.0 & 36.39 & 2.8 \\
\hline & 8 hours & HT-8Hrs & 6.41 & 302 & 0.0 & 37.70 & 0.0 \\
\hline
\end{tabular}
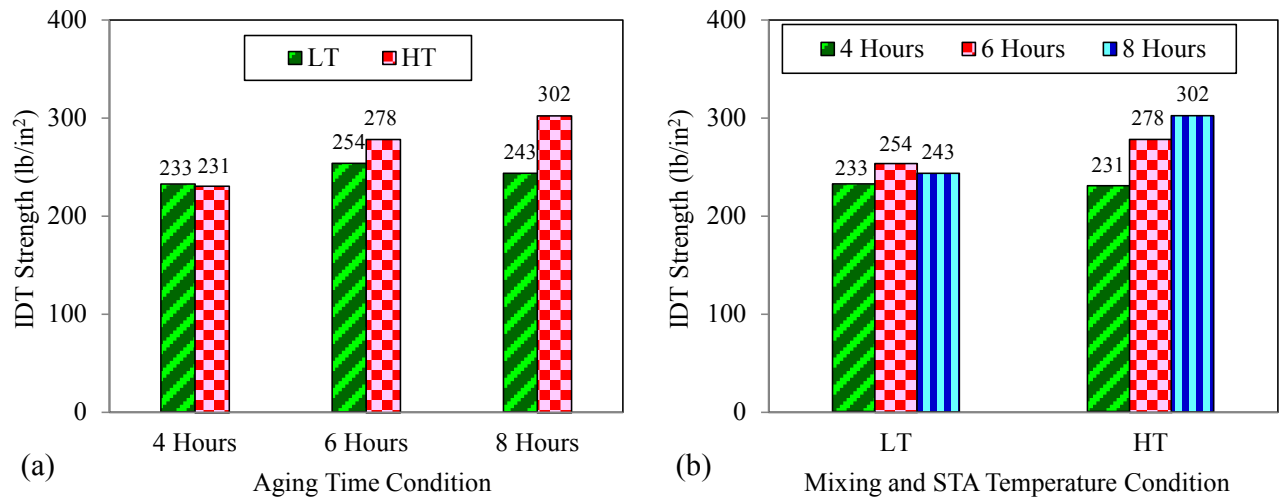

Fig. 5. IDT strength measured for both LT and HT conditions at 4, 6 and 8 hours STA times.

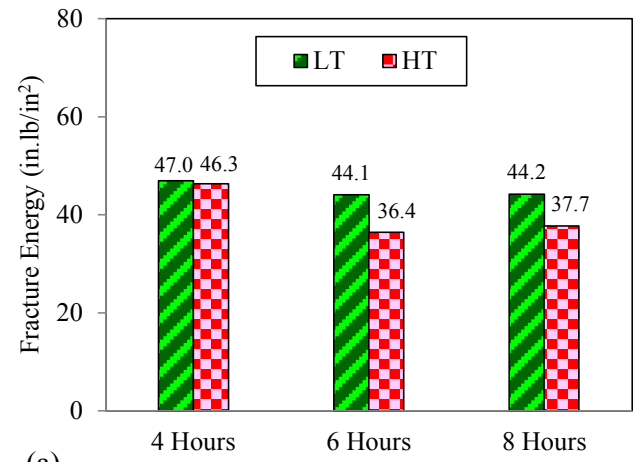

(a)

STA Time Condition

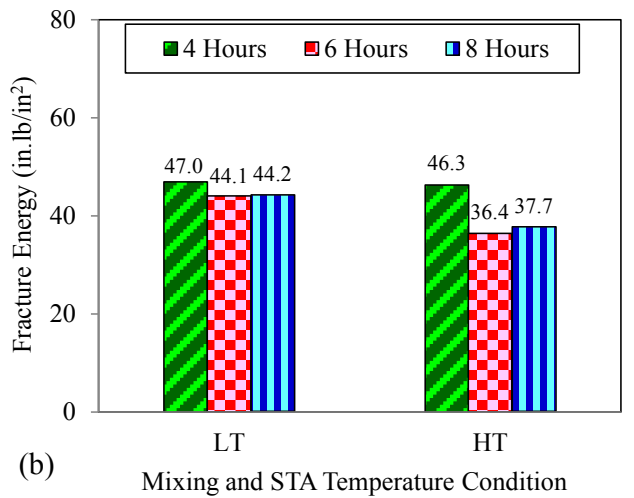

Fig. 6. Fracture Energy measured for both LT and HT conditions at 4, 6 and 8 hours STA times.

Table 8 and Table 9 include the statistical analysis results using paired t- test to evaluate the significance of elevated mixing and STA temperatures as well as the extended STA time on the changes in IDT strength and fracture energy respectively.

The results showed a significant increase in IDT strength and a significant decrease in fracture energy due to increasing the mixing and STA temperatures as well as the STA time. Keep in mind that these hypothesis analyses were conducted across all production temperature conditions and STA time cases. Statistical comparisons of individual cases were not possible due to sample size limitation (only one record for each individual case). 
Table 8: Statistical analysis of IDT strength results using paired t-test.

\begin{tabular}{|c|c|c|c|c|c|c|}
\hline \multirow{3}{*}{ Paired T-Test Results } & \multicolumn{6}{|c|}{ IDT Strength } \\
\hline & \multicolumn{2}{|c|}{$\begin{array}{l}\text { Effect of Mixing } \\
\text { and STA Temp. }\end{array}$} & \multicolumn{4}{|c|}{ Effect of STA Time } \\
\hline & $\mathbf{L T}$ & HT & $\begin{array}{c}4 \\
\text { Hours }\end{array}$ & $\begin{array}{c}6 \\
\text { Hours }\end{array}$ & $\begin{array}{c}4 \\
\text { Hours }\end{array}$ & $\begin{array}{c}8 \\
\text { Hours }\end{array}$ \\
\hline Mean, $\boldsymbol{\mu}$ & 243 & 270 & 232 & 266 & 232 & 273 \\
\hline Std Dev, $\sigma$ & 10.5 & 36.1 & 1.4 & 17.0 & 1.4 & 41.7 \\
\hline Degrees of Freedom, v & \multicolumn{2}{|c|}{2} & \multicolumn{2}{|c|}{2} & \multicolumn{2}{|c|}{2} \\
\hline t Sta. $\alpha / 2, v$ & \multicolumn{2}{|c|}{1.53} & \multicolumn{2}{|c|}{2.61} & \multicolumn{2}{|c|}{1.33} \\
\hline t Critical Two-Tail & \multicolumn{2}{|c|}{4.30} & \multicolumn{2}{|c|}{12.71} & \multicolumn{2}{|c|}{12.71} \\
\hline$P(T<=t)$ Two-Tail & \multicolumn{2}{|c|}{0.266} & \multicolumn{2}{|c|}{0.232} & \multicolumn{2}{|c|}{0.411} \\
\hline $\begin{array}{c}\text { Statistical Diff. Between } \\
\text { Sample Means } \\
\end{array}$ & \multicolumn{2}{|c|}{ SIGNIFICANT } & \multicolumn{2}{|c|}{ SIGNIFICANT } & \multicolumn{2}{|c|}{ SIGNIFICANT } \\
\hline
\end{tabular}

Table 9: Statistical analysis of IDT strength results using paired t-test.

\begin{tabular}{|c|c|c|c|c|c|c|}
\hline \multirow{3}{*}{ Paired T-Test Results } & \multicolumn{6}{|c|}{ Fracture Energy } \\
\hline & \multicolumn{2}{|c|}{$\begin{array}{l}\text { Effect of Mixing } \\
\text { and STA Temp. }\end{array}$} & \multicolumn{4}{|c|}{ Effect of STA Time } \\
\hline & $\mathbf{L T}$ & HT & $\begin{array}{c}4 \\
\text { Hours } \\
\end{array}$ & $\begin{array}{c}6 \\
\text { Hours } \\
\end{array}$ & $\begin{array}{c}4 \\
\text { Hours }\end{array}$ & $\begin{array}{c}8 \\
\text { Hours } \\
\end{array}$ \\
\hline Mean, $\mu$ & 45.1 & 40.1 & 46.6 & 40.2 & 46.6 & 41.0 \\
\hline Std Dev, $\sigma$ & 1.6 & 5.4 & 0.5 & 5.4 & 0.5 & 4.6 \\
\hline Degrees of Freedom, v & \multicolumn{2}{|c|}{2} & \multicolumn{2}{|c|}{1} & \multicolumn{2}{|c|}{1} \\
\hline t Sta. a/2,v & \multicolumn{2}{|c|}{2.28} & \multicolumn{2}{|c|}{1.82} & \multicolumn{2}{|c|}{1.94} \\
\hline t Critical Two-Tail & \multicolumn{2}{|c|}{4.30} & \multicolumn{2}{|c|}{12.71} & \multicolumn{2}{|c|}{12.71} \\
\hline $\mathbf{P}(\mathbf{T}<=t)$ Two-Tail & \multicolumn{2}{|c|}{0.150} & \multicolumn{2}{|c|}{0.319} & \multicolumn{2}{|c|}{0.304} \\
\hline $\begin{array}{c}\text { Statistical Diff. Between } \\
\text { Sample Means }\end{array}$ & \multicolumn{2}{|c|}{ SIGNIFICANT } & \multicolumn{2}{|c|}{ SIGNIFICANT } & \multicolumn{2}{|c|}{ SIGNIFICANT } \\
\hline
\end{tabular}

\section{Conclusions}

The objective of this research was to investigate how production (mixing and STA) temperatures and exposure time to elevated production temperatures affects aging and stiffening and properties of asphalt binders and asphalt mixtures. While thus far, most studies have focused on long term aging, this study focused only on the effects of short term aging conditions on the laboratory behavior of asphalt binder and mixtures. The study was conducted in two stages: asphalt binder stage and asphalt mixture stage. The following conclusions are made based on the study results:

1. Consistency results from the asphalt binder study showed that doubling the RTFO aging time from standard 85 minutes to 170 minutes caused the highest increase in 
viscosity and softening point compared the effect of the of increasing the RTFO aging temperature by $25^{\circ} \mathrm{F}$.

2. Stiffer binders seem more susceptible to elevated short term aging temperature and extended aging time when compared to softer binders.

3. The asphalt mixtures results using PG 64-22 asphalt binder showed in general that increasing the mixing and STA temperatures by $25^{\circ} \mathrm{F}$ changed the stiffness and strength of the mixtures. However, the results showed that the most significant changes in the $\left|E^{*}\right|$, IDT tensile strength and fracture energy occurred when the STA time was increased from 4 to 8 hours regardless of the mixing and STA temperatures.

4. Results from $\left|E^{*}\right|$ and IDT tests indicated that there is no significant effect of increasing the mixing and STA temperatures by $25^{\circ} \mathrm{F}$ on the $\left|E^{*}\right|$, IDT tensile strength and fracture energy values using a standard STA time of 4 hours.

5. More attentions should be paid into STA time including storage, transportation, and waiting time at the construction site as this can significantly alter the stiffness and the strength of asphalt mixtures.

6. Overall, the study recommends increasing the producing temperature by $25{ }^{\circ} \mathrm{F}$ during cold seasons or in cold regions to help achieving target compaction levels when using PG 64-22 or stiffer asphalt binders.

7. This recommendation may not be applied to other asphalt binders with PG grade that is softer than PG 64-22 asphalt binders unless it is proved by a similar laboratory study.

\section{References}

1. M.W. Mirza, and M.W. Witczak. Development of a global aging system for short and long term aging of asphalt cements. Journal of the Association of Asphalt Paving Technologists, vol. 64 (1995).

2. R.N. Hunter, A. Self, and J. Read. The shell bitumen handbook. London: ICE Publishing, pp. 744-747 (2015).

3. G.D. Airey, and S.K. Brown. Rheological performance of aged polymer modified bitumen. Journal of the Association of Asphalt Technologists, Volume. 67 (1998).

4. C.A. Bell, A.J. Weider, and M.J. Fellin. Laboratory aging of asphalt-aggregate mixtures: field validation. Strategic Highway Research Program, No. SHRP-A-390, National Research Council, Washington, DC (1994).

5. C.L. Monismith, R.G. Hicks, F.N. Finn, A.A. Tayebali, J.B. Sousa, J. Harvey, J.A. Deacon, T. Vinson, C. Bell, R. Terrel, and T. Scholz . Accelerated performance-related tests for asphalt-aggregate mixes and their use in mix design and analysis systems. Strategic Highway Research Program, No. SHRP-A-417, National Research Council, Washington, DC (1994).

6. R.C. Clark. Practical results of asphalt hardening on pavement life. Journal of the Association of Asphalt Paving Technologists, vol. 27, pp.196-208 (1958).

7. ASTM designation D2872-04. standard test method for effect of heat and air on a moving film of asphalt (rolling thin-film oven test).

8. ASTM designation D44-02. standard test method for viscosity determination of asphalt at elevated temperatures using a rotational viscometer.

9. ASTM designation D5-06. standard test method for penetration of bituminous materials.

10. ASTM designation D36/D36M. standard test method for softening point of bitumen (ring-and-ball apparatus). 
11. AASHTO designation TP62-07. standard method of test for determining dynamic modulus of hot mix asphalt concrete mixtures. AASHTO provisional standards, Washington, D.C., USA, 2007.

12. ASTM designation D6931-07. standard test method for indirect tensile (IDT) strength of bituminous mixture. 